\title{
On the origin of relativistic solar particle events: interplanetary transport modelling and radio emission
}

\author{
K.-L. Klein* ${ }^{\dagger}$ \\ LESIA (UMR 8109) and Station de Radioastronomie de Nançay, Observatoire de Paris, CNRS, \\ Meudon \\ E-mail: ludwig.klein@obspm.fr
}

\section{N. Agueda}

University of Barcelona, ICC-UB

E-mail: n. agueda@ub. edu

\section{R. Bütikofer}

Physikalisches Institut, University of Bern / HFSJG, Bern, Switzerland

E-mail: rolf.buetikofer@space.unibe.ch

\begin{abstract}
The highest energies of solar energetic nucleons detected in space or through gamma-ray emission in the solar atmosphere are in the GeV range. Where and how these particles are accelerated is still controversial. The candidate processes are related to magnetic reconnection in a flare or a coronal mass ejection (CME), and to the shock wave driven by a fast CME. We search for observational indications on the acceleration site, using the relative timing of radio emissions in the corona and of relativistic protons at the Earth. We model the particle transport under the effects of focussing by an average Archimedean magnetic field and of scattering by its fluctuations, and use radio time profiles as injection functions. The predicted time profiles of the intensity and anisotropy of relativistic protons at $1 \mathrm{AU}$ during the 20 Jan 2005 event are compared with neutron monitor observations. We compare the results with other events and discuss the nature of the prompt and delayed proton releases that have been identified in neutron monitor recordings of relativistic solar particle events.
\end{abstract}

The 34th International Cosmic Ray Conference,

30 July- 6 August, 2015

The Hague, The Netherlands

\footnotetext{
${ }^{*}$ Speaker.

†This work has received funding from the European Union's Horizon 2020 research and innovation programme under grant agreement No 637324.
} 


\section{Introduction}

Instabilities of the magnetic field in the corona create explosive releases of stored energy, which lead to the heating of plasma, to the acceleration of charged particles to energies that may by far exceed the average thermal energy of about $100 \mathrm{eV}$, and to the ejection of major parts of large-scale magnetic field structures. On certain occasions, charged particles are accelerated to relativistic energies. Nucleons penetrating into the Earth's atmosphere with energies exceeding $\sim 450 \mathrm{MeV}$ create secondaries that may reach the ground and can be detected by neutron monitors. These Ground Level Events (GLEs) reveal the highest energies that can be achieved during a solar energetic particle (SEP) event. They are a major and so far unsolved challenge to our understanding of particle acceleration in the solar corona.

GLEs are invariably associated with flares of high intensities in all spectral ranges of flarerelated electromagnetic emissions, notably gamma-rays, hard and soft $\mathrm{X}$-rays, and radio waves, as well as with fast coronal mass ejections (CMEs). These associations suggest that the particle acceleration is related with one or a combination of the acceleration processes thought to be at work in flares and CMEs: magnetic reconnection and large-scale shock waves.

Magnetic reconnection is supposed to act in two different situations: (1) In the early (impulsive) phase of the flare, i.e. the time interval between the flare onset and the peak of the soft X-ray emission, the process is thought to lead to plasma heating and to the acceleration of electrons and protons that produce the most intense gamma-ray, hard X-ray and microwave (radio waves at frequencies $v \geq 1 \mathrm{GHz}$, wavelengths $\lambda \leq 30 \mathrm{~cm}$ ) emission of the flare. (2) The early development of a CME is generally thought to comprise the formation of a flux rope and of current sheets in its aftermath, where magnetic reconnection is expected to occur over much longer duration than the impulsive phase [1]. Distinctive radiative signatures are notably long-lasting radio emissions, called type IV bursts, extending from centimetric-to-metric radio waves [2, 3, 4]. The actual particle acceleration process can be related to different ways to generate electric fields: direct electric fields in the reconnection region, the dynamical evolution of the current sheet due to tearing and coalescence of magnetic islands, waves and turbulence, betatron acceleration in retracting reconnected magnetic field lines, and shock waves that may be produced by the outflow jets from the reconnecting current sheets. Shock waves are also driven on much larger spatial scales by fast CMEs. They are likely candidates for the long-lasting SEP events at $\mathrm{MeV}$ and possibly higher energies.

While we observe relativistic particles, we are so far unable to model their acceleration in a fully consistent way. Acceleration models usually consider the motion of test particles in the electric and magnetic fields derived from MHD models. But when, as in the events of interest, the non-thermal particles carry a significant fraction of the energy released during the flare or conveyed to the CME, the particles will retro-act on the electromagnetic fields - a process that is not described by MHD. For this reason observational evidence on the acceleration regions is important to make progress in understanding the acceleration process at work.

We discuss in this contribution the timing of radiative signatures of particle acceleration and transport in the solar corona, using especially radio waves, with respect to the arrival of relativistic protons at the Earth inferred from neutron monitor observations and their inversion. We focus on the GLE of 20 Jan 2005, a prime example of a double-peaked proton time profile, which [5] 
established as a general feature of GLEs from eruptive flares in the western hemisphere. These authors argued that the first peak, which is short and displays a strong anisotropy [6], is missing in most GLEs. This time structure enables us to identify time relationships with the electromagnetic emission at the Sun, in an attempt to trace the particles at 1 AU back to their origin near the Sun (Sect. 2). We establish the connection by modelling the interplanetary particle transport under the effects of adiabatic focussing and pitch-angle scattering. The results for the $20 \mathrm{Jan} 2005$ GLE are compared in Sect. 3 with two other GLEs (29 Sep 1989, 14 Jul 2000), where the first relativistic proton pulse was missed by the neutron monitor network. We argue that our analysis supports a scenario of relativistic proton acceleration related to magnetic reconnection in the impulsive and post-impulsive evolution of an eruptive flare.

\section{The GLE of 20 January 2005}

\subsection{Observations: a brief overview}

The 20 Jan 2005 event has been extensively discussed in the literature. Here we summarise results from [8] and [7]. Figure 1 compares the intensity-time profiles of relativistic protons at two energies (Fig. 1.e), derived from the inversion of neutron monitor observations, with radio emissions from microwaves (Fig. 1.b) to km-waves (Fig. 1.d), and with soft X-rays (Fig. 1.a). The soft $\mathrm{X}$-rays trace the evolution of the flare-heated plasma in the low corona. The radio emissions are produced by relativistic electrons (hundreds of $\mathrm{keV}$ to some $\mathrm{MeV}$ ) in the low corona (microwaves; gyro synchrotron emission) and by electrons of probably some $\mathrm{keV}$ to some tens of $\mathrm{keV}$ (dm-to$\mathrm{km}$ wavelengths) between the corona and the Earth. Roughly speaking, the altitude of emission increases with decreasing frequency in the dynamic spectrograms (Fig. 1.b-d). The radiative signatures can be summarised as follows:

- The radio emission shows a similar distinction of two parts as the proton profiles. At frequencies between some tens of $\mathrm{MHz}$ (corona, about $1 \mathrm{R}_{\odot}$ above the photosphere) and some tens of $\mathrm{kHz}$ (vicinity of the Earth; Fig. 1.d) the radio emission consists of type III bursts. These bursts show that electrons accelerated in the corona escape into the interplanetary space. We presume that protons behave similarly. We shifted the proton profiles (Fig. 1.e) back in time by $216 \mathrm{~s}$, so that their first rise matches the prominent rise of the first group of type III bursts.

- Part 1 of the radio emission occurs during the impulsive phase, i.e. during the rise of the soft X-ray burst. Pion-decay gamma-rays at energies above $60 \mathrm{MeV}$, observed by the SONG experiment aboard the CORONAS mission, reveals the presence of protons with energies above $300 \mathrm{MeV}$ (see also [9]), starting at 06:45:30 UT. Relativistic protons were hence accelerated during the impulsive phase of the flare. From the radio observations, we conclude that these protons are able to escape into the interplanetary space. If the onset of the GLE at 06:50 UT is due to these protons, they must have travelled $12.5 \mathrm{~min}$, which implies a distance of $1.5 \mathrm{AU}$ at a speed of $0.98 c$ (rigidity $5 \mathrm{GV}$ ).

- The second peak of the relativistic proton profile at the Earth corresponds to the second part of the radio emission. The second microwave peak at $2.7 \mathrm{GHz}$, with only a weak counterpart above $10 \mathrm{GHz}$ (Fig. 1.b), shows that the relativistic electrons are now released into higher 

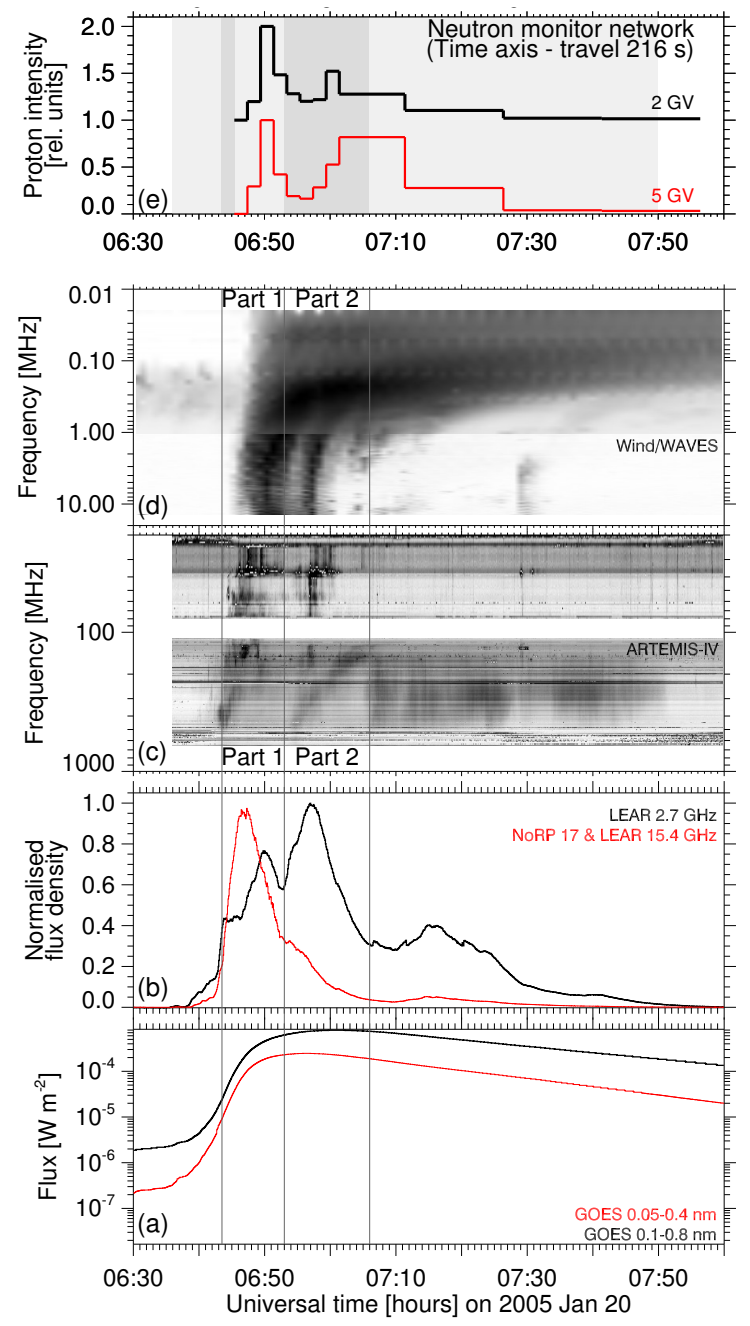

Figure 1: X-ray and radio emission and the relativistic proton profile of the 2005 Jan 20 event (adapted from [7]). From bottom to top: (a) soft X-rays $\lambda=0.1-0.8 \mathrm{~nm}$ (black line) and 0.05-0.4 nm (red line); (b) microwaves (black line: $2.7 \mathrm{GHz}$, red line: a combination of $17 \mathrm{GHz}$ (Nobeyama Radio Polarimeter) before and $15.4 \mathrm{GHz}$ (Learmonth RSTN station) after 06:55 UT); (c) dynamic radio spectrum at dm-m waves (ARTEMIS-IV, Univ. Athens; inverse colour scale; $1 \mathrm{~s}$ integration time); (d) decametre-kilometre wave radio emission (Wind/WAVES; inverse colour scale; 1 min integration); (e) proton flux time history at $2 \mathrm{GV}$ (black curve) and $5 \mathrm{GV}$ (red curve) rigidity (kinetic energy 1.27 and $4.15 \mathrm{GeV}$, respectively), time axis shifted back by $216 \mathrm{~s}$. The intervals delimited by vertical lines show the separation between the two parts of the GLE and of the radio emission, as discussed in the text.

structures, with weaker magnetic fields, than during the impulsive phase. At frequencies below $1000 \mathrm{MHz}$, the radio emission consists of a type IV burst with slow drift from high to low frequencies (Fig. 1.c), showing the release of non-thermal electrons into expanding magnetic structures in the course of the early development of the CME. The slowly drifting burst could be mistakenly identified as a type II burst, hence shock-accelerated electrons. But a detailed study of the spectrum [7] shows that this is not the case. This interpretation 
points to magnetic reconnection in the post-CME current sheet as the acceleration region of the radio-emitting electrons (see [4]), and tentatively also of the relativistic protons in the second part of the GLE.

The interplanetary travel path inferred from the above timing comparison is longer than the nominal Parker spiral. One way to explain this is that the travel of the particles is prolonged by pitch-angle scattering in the turbulent interplanetary magnetic field.

\subsection{Interplanetary transport modelling of particles accelerated at the Sun}

We use interplanetary transport simulations to model the propagation of solar relativistic particles from $2 \mathrm{R}_{\odot}$ to $1 \mathrm{AU}$. The model [10,11] solves the focussed transport equation [12], including the effects of adiabatic focussing by the outward decrease of the interplanetary magnetic field, and of pitch-angle scattering by magnetic fluctuations. The model assumes an Archimedean spiral magnetic flux tube connecting the Sun and the Earth, consistent with the solar wind speed observed in-situ. The free parameters of the model are the injection profile at the Sun and the interplanetary radial mean free path, $\lambda_{r}$, of the particles.

We assume that the injection profile at the Sun has the same time dependence as the microwave emission. We use the 15.4-17 GHz time profile (Fig. 1.b) as a proxy for the first solar release episode (06:41-06:53 UT), and the main peak of the $2.7 \mathrm{GHz}$ emission (06:53-07:05 UT) as a proxy of the second. Figure 2 shows the injection profile (top panel); the two injection components are colour-coded (red for the first one and blue for the second one). The first component peaks at 06:46 UT. The second component starts $11 \mathrm{~min}$ after the first and peaks at 06:56 UT. Since the goal of this study is to compare the characteristic time scales only, the two injection components are normalised and have the same peak amplitude.

Figure 2 (middle and bottom panel) shows the $5 \mathrm{GV}$ proton directional intensities expected at $1 \mathrm{AU}$ for two values of $\lambda_{r}$, with pitch angles $\alpha=0^{\circ}$ (propagating away from the Sun), $\alpha=90^{\circ}$ and $\alpha=180^{\circ}$ (propagating sunwards). The particle event produced by each injection component is colour-coded.

For $\lambda_{r}=2.0 \mathrm{AU}$ (middle panel), the onset of the event at $1 \mathrm{AU}$ is expected $\sim 8 \mathrm{~min}$ after injection. The field-aligned intensity $\left(\alpha \sim 0^{\circ}\right)$ is high due to adiabatic focussing. The intensity profile resembles the injection function. It shows a double-peaked structure, where the time delay between the pulses is the same as in the injection profile. The peak times of the two components at 1 AU agree with those observed by neutron monitors [5], as indicated by the arrows in Fig. 2. After 07:00 UT, both components merge and have a contribution to the intensities near Earth. Protons with $\alpha=90^{\circ}$ are expected to be observed at $1 \mathrm{AU} \sim 12$ min after the first release, with much smaller intensities. Intensities of sunward-propagating protons $\left(\alpha=180^{\circ}\right)$ are more than two orders of magnitude below those of protons with $\alpha=0^{\circ}$, i.e. their intensities are below the values plotted in this panel.

Thus, under these assumptions, the timing of the relativistic proton event at $1 \mathrm{AU}$ is consistent with the timing of the neutron monitor observations, suggesting that the two parts of the radio event are related to the two episodes of acceleration of the GLE. But while this scenario provides an explanation for the first anisotropic pulse of the GLE, the predicted pitch-angle distribution is inconsistent with the second, more isotropic, pulse. 


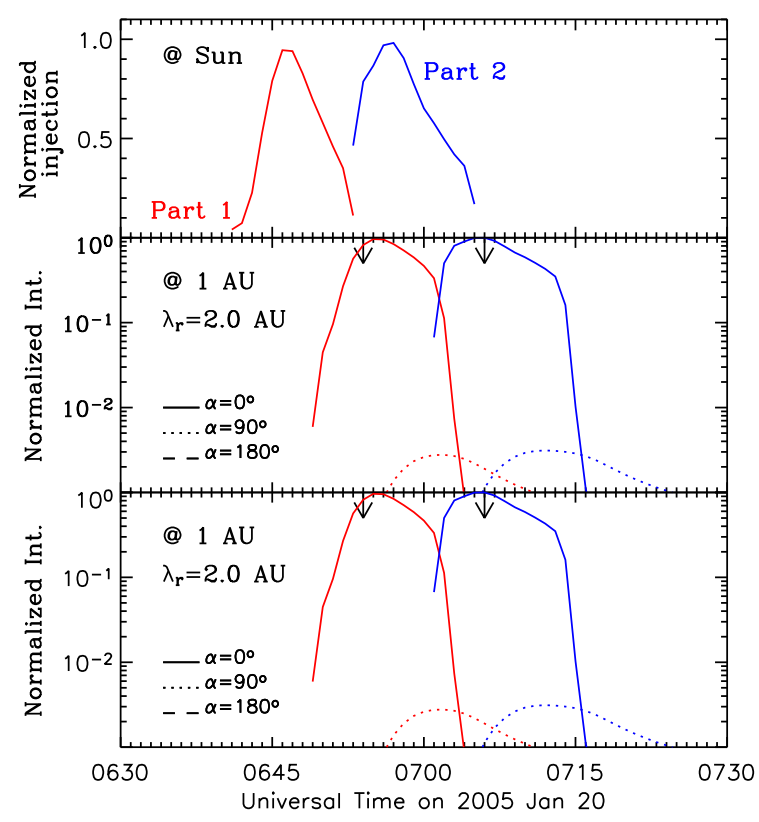

Figure 2: From top to bottom: Two-component injection profile assumed at the Sun based on the microwave emission at $\sim 15 \mathrm{GHz}$ (red) and $2.7 \mathrm{GHz}$ (blue), respectively; see text for details. Directional $5 \mathrm{GV}$ proton intensities at $1 \mathrm{AU}$ for three different pitch-angles: $0^{\circ}$ (solid), $90^{\circ}$ (dotted) and $180^{\circ}$ (dashed). The intensity profiles associated with each injection component are colour-coded (red for the first component, blue for the second one). The vertical arrows mark the time of the two GLE pulses according to [5]. The interplanetary mean free path is $\lambda_{r}=2.0 \mathrm{AU}$ and $\lambda_{r}=0.5 \mathrm{AU}$, respectively.

One of the effects that would contribute to isotropise the pitch-angle distributions is stronger interplanetary scattering. The bottom panel of Fig. 2 shows the results for $\lambda_{r}=0.5$ AU. The intensity time profile for $\alpha=0^{\circ}$ resembles the injection function in the sense that it shows a doublepeaked structure. The peaks occur several minutes after the maxima of the GLE pulses, while the time delay between the pulses is the same as in the injection profile. Since protons are more frequently scattered than for $\lambda_{r}=2.0 \mathrm{AU}$, the pitch-angle distribution is more isotropic, as shown by the increased proton intensities for larger values of $\alpha$. Sunward-streaming protons are expected to be observed at $1 \mathrm{AU}$ about $15 \mathrm{~min}$ after the first release. Thus with a smaller proton mean free path the second pulse is more isotropic, but the timing of the peaks then disagrees with the observations.

\section{Discussion and Conclusion}

We applied a model of focussed interplanetary transport of relativistic protons accelerated in the corona to understand the intensity and anisotropy of the protons measured by neutron monitors on the Earth. The two proton releases identified during the GLE on 20 Jan 2005 could in this way be related to the times when electrons were accelerated at the Sun, as revealed by their radio emission. A small amount of interplanetary pitch-angle scattering, described by a mean free path $\lambda_{r}=2 \mathrm{AU}$, is consistent with the timing of the GLE and the solar radio emission. It is also consistent with the high anisotropy during the first GLE pulse. The model results confirm the close association of the 
early GLE pulse with the impulsive phase of the flare. Since relativistic protons were observed in the solar atmosphere, and since radio signatures showed that at that time particles could escape into the interplanetary space, the transport modelling supports the scenario of a common acceleration of interacting and escaping relativistic protons. This acceleration is related to magnetic reconnection during the development of the flare and CME, in accordance with earlier conclusions [9, 8].

But the interplanetary transport model predicts a more anisotropic second pulse than observed. Enhancing the scattering would provide a better account of the anisotropy, but predict too late a particle arrival at $1 \mathrm{AU}$. A particle distribution released farther away from the Sun would suffer less focussing, and would be observed more isotropically at $1 \mathrm{AU}$. While the microwave spectrum gives evidence that the second part of the radio event is produced at greater altitude than the impulsive phase emission, the difference is a fraction of a solar radius, which is likely insufficient to produce a detectable difference in the pitch-angle distributions at 1 AU. McCracken et al. [9] used the argument to support the idea that the second relativistic proton pulse was due to acceleration at the CME shock far above the flaring active region. The CME front was at a heliocentric distance of about $4.5 \mathrm{R}_{\odot}$ at 6:54 UT [8]. But even such a height difference does not produce a detectable effect in the anisotropy of the GLE: our modelling shows that there is no significant difference beyond about 0.2 AU. However, back-reflection of protons from the first release at magnetic field structures beyond Earth would also reduce the anisotropy of the second pulse. It is plausible that this actually happened on 20 Jan 2005, because, like during most GLEs [13], the interplanetary medium was strongly disturbed by CMEs that occurred earlier, and which could constitute magnetic barriers beyond $1 \mathrm{AU}$ (see [14]).

We therefore conclude that the basic scenario of a coronal proton acceleration during the impulsive flare phase for the first pulse, and in the post-eruptive current sheet for the second, remains an attractive interpretation that could be a framework for understanding the time profiles of GLEs as seen by neutron monitors in general. The systematic study of GLEs [5, 6] showed that two peaks like on 20 Jan 2005 are typical of western events, with a presumable direct magnetic connection to the Earth. In the other, more numerous events, a single peak was seen. The authors suggest that this single peak is similar to the second pulse of the 20 Jan 2005 event, and that the first pulse is not observed when there is no direct magnetic connection to the site of the impulsive flare.

Our earlier analyses of two GLEs with single pulses, on 29 Sep 1989 [15] and 14 Jul 2000 [16], showed that the onsets of the GLEs were indeed delayed with respect to the expected arrival of the first relativistic protons accelerated during the impulsive flare phase. Both events were associated with flares about $40^{\circ}$ away from the longitude that is nominally connected to the Earth. In both events the onset of the GLE could be traced back to metre-wave type IV bursts, similar to part 2 of the 20 Jan 2005 GLE. This substantiates the argument that relativistic protons observed during GLEs are accelerated in flares and in the aftermath of a CME, presumably in relation with magnetic reconnection in the post-CME current sheet. How protons can be accelerated to relativistic energies in these current sheets, which are higher in the corona than typical acceleration regions in the impulsive flare phase, is not clear, and will need new modelling efforts. But the chain of remote sensing observations of the corona, in situ particle measurements at the Earth, and interplanetary particle transport modelling in between appears as a powerful tool to bring new observational constraints. 


\section{References}

[1] T. G. Forbes, J. A. Linker, J. Chen, et al., CME theory and models, Space Sci. Rev. 123 (2006) 251-302.

[2] S. W. Kahler and A. J. Hundhausen, The magnetic topology of solar coronal structures following mass ejections, J. Geophys. Res. 97 (1992) 1619-1631.

[3] K.-L. Klein and Z. Mouradian, The dynamics of an erupting prominence, Astron. Astrophys. 381 (2002) 683-693.

[4] P. Démoulin, A. Vourlidas, M. Pick, and A. Bouteille, Initiation and development of the white-light and radio coronal mass ejection on 2001 April 15, Astrophys. J. 750 (2012) 147.

[5] H. Moraal and K. G. McCracken, The time structure of ground level enhancements in solar cycle 23, Space Sci. Rev. 171 (2012) 85-95.

[6] K. G. McCracken, H. Moraal, and M. A. Shea, The high-energy impulsive ground-level enhancement, Astrophys. J. 761 (2012) 101.

[7] K.-L. Klein, S. Masson, C. Bouratzis, V. Grechnev, A. Hillaris, and P. Preka-Papadema, The relativistic solar particle event of 2005 January 20: origin of delayed particle acceleration, Astron. Astrophys. 572 (2014) A4.

[8] S. Masson, K.-L. Klein, R. Bütikofer, E. O. Flückiger, V. Kurt, B. Yushkov, and S. Krucker, Acceleration of relativistic protons during the 20 January 2005 flare and CME, Solar Phys. 257 (2009) 305-322.

[9] K. G. McCracken, H. Moraal, and P. H. Stoker, Investigation of the multiple-component structure of the 20 January 2005 cosmic ray ground level enhancement, J. Geophys. Res. 113 (2008), no. A12 12101.

[10] N. Agueda, R. Vainio, D. Lario, and B. Sanahuja, Injection and interplanetary transport of near-relativistic electrons: Modeling the impulsive event on 2000 May 1, Astrophys. J. 675 (2008) 1601-1613.

[11] N. Agueda, K.-L. Klein, N. Vilmer, et al., Release timescales of solar energetic particles in the low corona, Astron. Astrophys. $\mathbf{5 7 0}$ (2014) A5.

[12] E. C. Roelof, Propagation of solar cosmic rays in the interplanetary magnetic field, in Lectures in High-Energy Astrophysics (H. Ögelmann and J. Wayland, eds.), pp. 111-136, NASA SP-199, 1969; ${ }^{1}$.

[13] S. Masson, P. Démoulin, S. Dasso, and K.-L. Klein, The interplanetary magnetic structure that guides solar relativistic particles, Astron. Astrophys. 538 (2012) A32.

[14] A. Sáiz, D. Ruffolo, J. W. Bieber, P. Evenson, and R. Pyle, Anisotropy signatures of solar energetic particle transport in a closed interplanetary magnetic loop, Astrophys. J. 672 (Jan., 2008) 650-658.

[15] K.-L. Klein, E. L. Chupp, G. Trottet, A. Magun, P. P. Dunphy, E. Rieger, and S. Urpo, Flare-associated energetic particles in the corona and at 1 AU, Astron. Astrophys. 348 (1999) 271-285.

[16] K.-L. Klein, G. Trottet, P. Lantos, and J.-P. Delaboudinière, Coronal electron acceleration and relativistic proton production during the 14 July 2000 flare and CME, Astron. Astrophys. 373 (2001) 1073-1082. Erratum: Astron. Astrophys. 377 (2001) 687.

\footnotetext{
${ }^{1}$ electronic version at ntrs.nasa.gov/archive/nasa/casi.ntrs.nasa.gov/19690020274.pdf
} 